\title{
Microwave Spectrum and Barrier to Internal Rotation of 1-Chloro-2-butyne
}

\author{
V. M. STOLWIJK AND B. P. VAN EIJCK \\ Department of Structural Chemistry, University of Utrecht, Padualaan 8, \\ $3584 \mathrm{CH}$ Utrecht, The Netherlands
}

\begin{abstract}
Rotational transitions of the $\mu_{a}$ and $\mu_{b}$ type have been identified with microwave-microwave double resonance measurements for 1-chloro-2-butyne in the ground vibrational state. In the first excited state of the methyl torsion only $\mu_{a}$-type transitions have been identified. The $A$-type transitions of the ground vibrational state can be described perfectly by the rigid rotor approximation with centrifugal corrections. Using the internal axis method the barrier to internal rotation was determined from the $A, E$ splittings: $V_{3}=10.05 \pm 0.09 \mathrm{~cm}^{-1}$. A model which allowed for geometry relaxation upon internal rotation was used to fit one set of parameters to the transition frequencies of both ground state and first excited torsional state. The sixfold contribution to the barrier was found to be negligible: $V_{6}=-0.4 \pm 0.3 \mathrm{~cm}^{-1}$. @ 1987 Academic Press, Inc.
\end{abstract}

\section{INTRODUCTION}

Many barriers to internal rotation have been determined by microwave spectroscopy. However, the number of publications $(1-7)$ where a low threefold barrier (say, $V_{3}$ $<30 \mathrm{~cm}^{-1}$ ) has been studied in asymmetric molecules is not large. In some of these cases $V_{3}$ and $V_{6}$ become comparable in magnitude and two sets of values for these parameters are found to be compatible with the observations: $V_{3}=10$ or $21 \mathrm{~cm}^{-1}$ in trans-methyl nitrite (4) and $V_{3}=15.8$ or $16.6 \mathrm{~cm}^{-1}$ in meta-fluorotoluene (3). Differences between observed and calculated frequencies were sometimes considerable, which has been ascribed to possible interactions of the methyl torsion with the other molecular vibrations. For molecules containing the $\mathrm{CH}_{3}-\mathrm{N}=\mathrm{C}=$ fragment (2), Koput has recently taken into account the $\mathrm{CNC}$ bending mode and determined effective barriers of $20.6 \pm 0.1 \mathrm{~cm}^{-1}$ for methyl isocyanate (6) and $1.7 \pm 0.4 \mathrm{~cm}^{-1}$ for methyl isothiocyanate (7). All these studies used free internal rotor functions as the basis for the calculations. Alternatively, the internal axis method (IAM) has been used for acetamide (5) to obtain a barrier of $24.63 \pm 0.03 \mathrm{~cm}^{-1}$.

In an early study of 1-chloro-2-butyne, $\mathrm{CH}_{3}-\mathrm{C} \equiv \mathrm{C}-\mathrm{CH}_{2} \mathrm{Cl}$, Laurie and Lide (I) determined effective rotational constants for the torsional $A$ ground state from $\mu_{a}$-type transitions. Comparison with the model parameters enabled these investigators to establish a conservative upper limit of about $30 \mathrm{~cm}^{-1}$ for $V_{3}$. We decided to try to determine this barrier height more accurately by searching for $\mu_{b}$-type transitions and $A, E$ splittings. This work should also be of interest for comparison of the barrier height with symmetric molecules with a triple bond: $\mathrm{CH}_{3}-\mathrm{C} \equiv \mathrm{C}-\mathrm{CD}_{3}\left(V_{3}=5.62 \pm 0.16\right.$ $\left.\mathrm{cm}^{-1}(8)\right), \mathrm{CF}_{3}-\mathrm{C} \equiv \mathrm{C}-\mathrm{CH}_{3}\left(V_{3}<100 \mathrm{~cm}^{-1}(9)\right), \mathrm{CH}_{3}-\mathrm{C} \equiv \mathrm{C}-\mathrm{SiH}_{3}\left(V_{3}=3.77\right.$ $\left.\pm 0.70 \mathrm{~cm}^{-1}(10)\right)$. Moreover, we were interested to see how well the IAM would 
perform in the region of low barriers. The work on acetamide (5), where large discrepancies between observed and calculated frequencies were encountered, is not encouraging. On the other hand, trial calculations showed that the IAM should work well down to quite low barriers, provided, of course, that the real molecule is well described by the model with the $\mathrm{CH}_{3}$ torsion being the only internal degree of freedom.

An indication that this model may be successful for $\mathrm{CH}_{3}-\mathrm{C} \equiv \mathrm{C}-\mathrm{CH}_{2} \mathrm{Cl}$ is given by an infrared study down to $45 \mathrm{~cm}^{-1}$, where the lowest vibrational absorption was found at $119 \mathrm{~cm}^{-1}(11)$. All of the normal vibrational modes but the $\mathrm{CH}_{3}$ torsion were assigned. Some of the characteristics of the infrared spectrum could be explained by assuming free internal rotation.

\section{EXPERIMENTAL DETAILS}

The $\mathrm{CH}_{3} \mathrm{CCCH}_{2} \mathrm{Cl}$ was provided by Professor $\mathrm{L}$. Brandsma of the State University of Utrecht. It was used after distillation. At room temperature the compound is not stable (after 2 days the clear, colorless liquid becomes troubled and yellow), so the stock was kept at liquid nitrogen temperature. The measurements were made at room temperature and a gas pressure of about $0.05 \mathrm{~mm} \mathrm{Hg}$, with occasional renewal of the gas sample. Because one of the major problems in identifying the microwave spectrum of this type of molecules is the very dense Stark spectrum, the microwave-microwave double resonance (MW-MW DR) technique was used for identification. A 9-m $K$ band cell without Stark septum was used. The accuracy of the measurements is about $0.5 \mathrm{MHz}$ for frequencies only measured from pump settings and better than $0.1 \mathrm{MHz}$ for detection frequencies.

After identification of the ground vibrational state by these measurements the Stark spectrum $(800 \mathrm{~V} / \mathrm{cm})$ was recorded with a conventional $11-\mathrm{kHz}$ Stark-modulated spectrometer from 26.5 to $39.0 \mathrm{GHz}$ and in a few areas between 39.0 and $43.0 \mathrm{GHz}$.

\section{RESULTS}

\section{a. The Ground Vibrational State}

The $J=10 \leftarrow 9\left(K_{-1} \leqslant 2\right)$ transition frequencies reported by Laurie and Lide (l) were reproduced easily by MW-MW DR using the $J=9 \leftarrow 8$ transitions as pump. Because of the good correspondence, the other published values were used in further calculations too, an exception being made for the $K_{-1} \geqslant 3$ transitions. In these regions, even in the MW-MW DR measurements, too many lines were found to make a reliable assignment possible in this premature stage of the experiment. By means of a rigid rotor fit with centrifugal corrections other frequencies were predicted. The $a$ type lines were easily found; they showed no splitting due to ${ }^{35} \mathrm{Cl}$-quadrupole coupling. These data cannot give a well-determined rotational constant $A$, so the prediction of the $b$-type lines was thought to be pretty uncertain. Moreover, calculations based on quadrupole coupling constants taken from Ref. (12) predicted that these lines would be split into four components, thus making them rather weak. Much effort was spent on searching for quartets with the predicted structure (intervals of successively 1,6 , and $1 \mathrm{MHz}$ ). Eventually, for a pump setting at the $9_{09} \leftarrow 8_{08}$ frequency, two such quartets were found that were identified as the $9_{19} \leftarrow 8_{08}$ and $10_{1,10} \leftarrow 9_{09}$ transitions. Surprisingly, the mean frequencies had the same values as predicted by the above 
mentioned semirigid rotor fit. Now, other $b$-type transitions ( $P$ - and $Q$-type) could be predicted and searched for. Some were hard to find, because these lines are very weak and very sensitive to the pump setting. Finally, some isolated lines from the Stark spectrum could be reliably assigned, too. The resulting parameters of the semirigid rotor fit are given in Table I. The rms deviation of this fit was $0.17 \mathrm{MHz}$. The data did not allow a reliable determination of the quadrupole coupling constants, so for the $b$-type lines the averaged frequencies were used throughout.

To find the $E$-type transitions the same data were used in IAM calculations, with various values for $V_{3}$. In these calculations basis functions expi $\alpha(3 \mathrm{k}+\sigma)$, with $k$ ranging from -6 to +6 , were used, and in the Van Vleck transformation the summation over the excited torsional states was carried out up to $v=4(13)$. The $a$-type $K_{-1}=0$ transitions were the least sensitive to $V_{3}$, and these were found first. Then $K_{-1}=1$ transitions could be identified too, but no $E$-type $K_{-1} \geqslant 2$ transitions could be assigned reliably due to the crowding of even the double resonance spectrum. In the calculations the $V_{6}$ contribution to the torsional potential energy was arbitrarily set to zero. The resulting parameters are given in the second column of Table II, and the observed frequencies are collected in Table III. It is seen that, contrary to what was found for acetamide (5), the fit is quite good, even for this very low barrier (reduced barrier height $\mathrm{s}=0.776)$. Comparison between the obtained value of $\vartheta\left(19.6^{\circ}\right)$ and the value of the model $\left(20.6^{\circ}\right)$ shows a small tilt of the methyl group away from the $\mathrm{Cl}$ atom. Unfortunately, a correlation between $A$ and $I_{\alpha}$ is responsible for the relatively large uncertainties in these parameters.

\section{b. The First Excited Torsional State}

Using the parameters of Table II frequencies were predicted for the first excited state of the methyl torsion. It was found that the $A$-type transitions $J+1_{0, J+1} \leftarrow J_{0, J}$

TABLE I

Effective Rigid Rotor Constants and Centrifugal Distortion Parameters of $\mathrm{CH}_{3} \mathrm{CCCH}_{2} \mathrm{Cl}$ in the $A$-Type Ground Vibrational State $\left(I^{r}\right)$

\begin{tabular}{|c|c|c|c|c|}
\hline & & Mode1 (1) & $\operatorname{Ref} .(1)$ & Th1s work. \\
\hline A & $\left(\mathrm{MHz}^{2}\right)$ & 15925 & 17631 & $17615.77 \quad(11)$ \\
\hline B & $"$ & 1492 & 1490.548 (5) & 1490.582 \\
\hline c & $"$ & 1388 & $1385.061(5)$ & 1385.040 \\
\hline$\Delta_{\mathrm{J}}$ & $(\mathrm{kHz})$ & & $0.40 \quad(4)$ & 0.42 \\
\hline$\Delta_{J K}$ & $"$ & & $-28.4 \quad(20)$ & -27.7 \\
\hline$\Delta_{K}$ & $"$ & & & (29) \\
\hline$\delta_{J}$ & " & & & $0.062 \quad(3)$ \\
\hline$\delta_{\mathrm{K}}$ & $"$ & & & 5.2 \\
\hline s & $(M \mathrm{M} z)$ & & & 0.17 \\
\hline $\mathrm{N}$ & & & 23 & 58 \\
\hline$J_{\text {max }}$ & & & 10 & 22 \\
\hline
\end{tabular}

$s$ denotes the root mean square deviation and $N$ the number of transition frequencies used. 
TABLE II

Molecular Parameters of $\mathrm{CH}_{3} \mathrm{CCCH}_{2} \mathrm{Cl}$ in the Ground Vibrational State and $v=0,1$

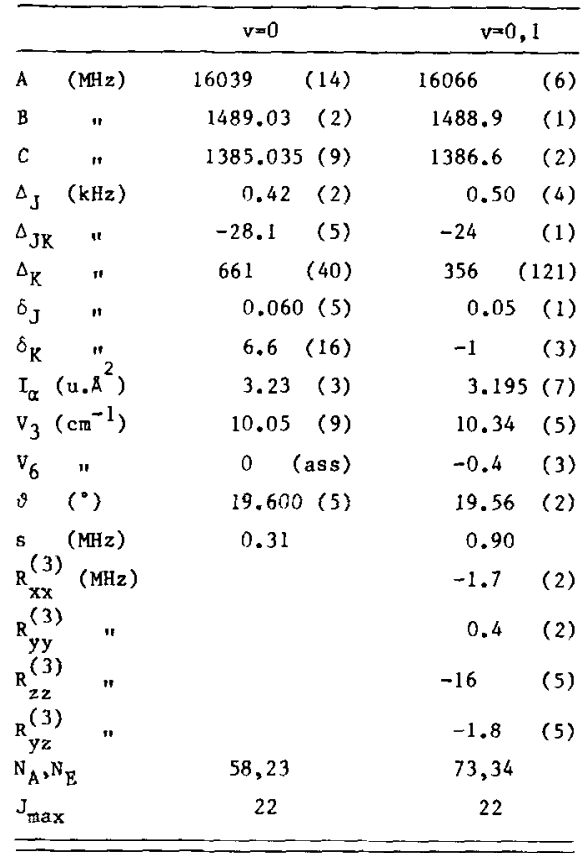

$\mathrm{I}_{\alpha}$ Moment of Inertia of the $\mathrm{CH}_{3}$ group about the Internal rotation axis.

$\vartheta$ The angle between the a principal axis and the internal rotation axis.

s Root mean square deviation.

N Number of lines used.

and $J+1_{1, J+1} \leftarrow J_{1, J}$ should be almost coincident. Furthermore, both $A$ - and $E$-type transitions with $K_{-1} \geqslant 3$ cluster in the same regions as in the ground vibrational state, thus making the analysis of these lines virtually impossible.

In the Stark spectrum relatively strong lines are present about $10 \mathrm{MHz}$ away from the calculated values of the $A$-type transitions $\Delta J=1, K_{-1}=0(9 \leqslant J \leqslant 14)$. Testing the three lowest transitions by MW-MW DR measurements the signal frequencies exhibited a doublet structure not resolved in the Stark measurements, probably caused by the almost overlapping $\Delta J=1, K_{-1}=1, K_{+1}=J$ transitions. The predicted frequencies of the corresponding $E$-type transitions were very near to lines in the Stark spectrum. These identifications were accepted only when no other lines were present within $10 \mathrm{MHz}$ or when the assignment could be verified by MW-MW DR. A few more $K_{-1} \neq 0$ lines could be assigned by MW-MW DR.

The number of identified transitions was too small to obtain a well-determined set of molecular parameters for the first excited torsional state. However, combination with the ground state data allowed us to determine parameters such as $V_{6}$ that cannot be found from the study of either torsional state by itself. The relevant theory and 
TABLE III

Ground State Transition Frequencies and $A, E$ Splittings of $\mathrm{CH}_{3} \mathrm{CCCH}_{2} \mathrm{Cl}(\mathrm{MHz})^{\mathrm{b}}$

\begin{tabular}{|c|c|c|c|c|c|c|c|c|c|c|}
\hline \multicolumn{6}{|c|}{ Transition } & \multirow{2}{*}{$\frac{\nu_{A}}{20491.70(\mathrm{~L})}$} & \multirow{2}{*}{$\frac{\Delta v_{\mathrm{A}}^{\mathrm{a}}}{-0.15}$} & \multirow[t]{2}{*}{${ }^{v_{E}}$} & \multirow[t]{2}{*}{$\Delta v_{E}$} & \multirow[t]{2}{*}{${ }^{v_{A}}{ }^{-v_{E}}$} \\
\hline 7 & 1 & 6 & +6 & 1 & 5 & & & & & \\
\hline 7 & 1 & 7 & $\leftarrow$ & 1 & 6 & $19753.23 \mathrm{n}$ & 0.03 & & & \\
\hline 8 & 0 & 8 & 4 & 0 & 7 & 22960.93 & -0.03 & & & \\
\hline 8 & 1 & 8 & +7 & 1 & 7 & 22572.56 & -0.06 & & & \\
\hline 8 & 1 & 7 & 47 & 1 & 6 & $23416.04 "$ & -0.01 & & & \\
\hline 8 & 2 & 7 & $\leftarrow$ & 2 & 6 & 22999.59 " & 0.03 & & & \\
\hline 8 & 2 & 6 & $\leftarrow 7$ & 2 & 5 & $23042.71 "$ & 0.12 & & & \\
\hline 9 & 0 & 9 & $\leftarrow 8$ & 0 & 8 & $25817.70 "$ & 0.04 & 25521.1 & 0.4 & 296.6 \\
\hline 9 & 1 & 9 & + & 0 & 8 & 39754.50 & -0.36 & 40747.38 & -1.00 & -992.88 \\
\hline 9 & 1 & 9 & $\leftarrow$ & 1 & 8 & $25390.67(\mathrm{~L})$ & 0.03 & & & \\
\hline 9 & 1 & 8 & $\leftarrow$ & 1 & 7 & $26339.34 \mathrm{n}$ & -0.02 & & & \\
\hline 9 & 2 & 8 & $\leftarrow$ & 2 & 7 & $25872.05 \|$ & 0.08 & & & \\
\hline 9 & 2 & 7 & + & 2 & 6 & 25933.76 " & 0.01 & & & \\
\hline 10 & 0 & 10 & $\leftarrow$ & 0 & 9 & 28669.96 & 0.01 & 28376.04 & 0.16 & 293.92 \\
\hline 10 & 1 & 10 & + & 0 & 9 & 42144.50 & -0.34 & & & \\
\hline 10 & 1 & 10 & $\leftarrow$ & 1 & 9 & 28207.61 & 0.15 & 28136.70 & 0.34 & 70.91 \\
\hline 10 & 1 & 9 & +9 & $i$ & 8 & 29261.28 & -0.03 & 29416.71 & 0.58 & -155.43 \\
\hline 10 & 2 & 9 & +9 & 2 & 8 & 28743.74 & 0.04 & & & \\
\hline 10 & 2 & 8 & $\leftarrow$ & 2 & 7 & 28828.27 & 0.08 & & & \\
\hline 10 & 3 & 8 & +9 & 3 & 7 & 28770.48 & 0.07 & & & \\
\hline 10 & 3 & 7 & +9 & 3 & 6 & 28771.71 & 0.09 & & & \\
\hline 10 & 4 & & +9 & 4 & & 28769.85 & 0.17 & & & \\
\hline 11 & 0 & 11 & +10 & 0 & 10 & 31517.05 & 0.12 & 31231.28 & 0.10 & 285.77 \\
\hline 11 & 1 & 10 & $\leftarrow 10$ & 1 & 9 & 32181.63 & 0.02 & 32309.80 & 0.08 & -128.17 \\
\hline 11 & 1 & 11 & +10 & 1 & 10 & 31023.70 & -0.13 & 30946.51 & 0.59 & 77.19 \\
\hline 11 & 2 & 10 & $\leftarrow 10$ & 2 & 9 & 31614.42 & 0.06 & & & \\
\hline 11 & 2 & 9 & +10 & 2 & 8 & 31726.77 & 0.14 & & & \\
\hline 11 & 3 & 9 & +10 & 3 & 8 & 31649.14 & 0.25 & & & \\
\hline l] & 3 & 8 & +10 & 3 & 7 & 31651.24 & 0.17 & & & \\
\hline 11 & 4 & & +10 & 4 & & 31647.78 & 0.18 & & & \\
\hline 11 & 1 & in & $\leftarrow 11$ & 0 & 11 & & & 23859.2 & 0.2 & \\
\hline 12 & 0 & 12 & +11 & 0 & 11 & $34358.86(\mathrm{~s})$ & 0.08 & 34085.86 & 0.12 & 273.00 \\
\hline 12 & 1 & 12 & $* 11$ & 1 & 11 & $33837.80 "$ & 0.25 & $33756.63(\mathrm{~s})$ & 0.42 & 81.17 \\
\hline 12 & 1 & 11 & +11 & 1 & 10 & 35100.28 & 0.05 & $35193.02 \%$ & 0.43 & -92.74 \\
\hline 12 & 2 & 10 & +11 & 2 & 9 & 34629.71 & 0.04 & & & \\
\hline 12 & 2 & 11 & +11 & 2 & 10 & 34484.17 & -0.04 & & & \\
\hline 13 & 0 & 13 & +12 & 0 & 12 & 37194.92 & -0.07 & 36939.00 & -0.07 & 255.92 \\
\hline 13 & 0 & 13 & +12 & 1 & 12 & 24735.0 & 0.21 & 22566.2 & 0.27 & 2168.80 \\
\hline 13 & 1 & 13 & $\leftarrow 12$ & 1 & 12 & 36651.17 & -0.07 & 36566.32 & 0.32 & 84.85 \\
\hline 13 & 1 & 12 & +12 & 1 & 11 & $38017.09(\mathrm{~s})$ & 0.02 & 38067.80 & 0.91 & -50.71 \\
\hline 13 & 2 & 12 & $\leftarrow 12$ & 2 & 11 & 37352.53 & 0.12 & & & \\
\hline 13 & 2 & 11 & $\leftarrow 12$ & 2 & 10 & $37537.10 \%$ & -0.00 & & & \\
\hline 13 & 1 & 12 & +13 & 0 & 13 & & & 26096.5 & 0.1 & \\
\hline 14 & 0 & 14 & +13 & 0 & 13 & 40025.01 & -0.19 & 39789.42 & -0.16 & 235.59 \\
\hline 14 & 0 & 14 & 413 & 1 & 13 & & & 25790.2 & -1.1 & \\
\hline 14 & 1 & 14 & -13 & 1 & 13 & $39462.86(\mathrm{~s})$ & -0.21 & 39375.53 & 0.16 & 87.33 \\
\hline 14 & 1 & 13 & +13 & 1 & 12 & 40931.73 & 0.06 & 40935.81 & 0.62 & -4.08 \\
\hline 14 & 2 & 12 & +13 & 2 & 11 & $40449.52(\mathrm{~S})$ & -0.38 & & & \\
\hline 14 & 2 & 13 & +13 & 2 & 12 & $40219.84 "$ & 0.08 & & & \\
\hline 14 & l & 13 & +14 & 0 & 14 & 22408.4 & 0.4 & 27243.9 & -0.10 & -4835.5 \\
\hline 15 & 0 & 15 & +14 & 0 & 14 & 42848.53 & -0.10 & 42636.08 & -0.02 & 212.45 \\
\hline 15 & 1 & 14 & $\leftarrow 15$ & 0 & 15 & 23405.0 & -0.5 & & & \\
\hline 17 & 1 & 16 & $\leftarrow 17$ & 0 & 17 & 25677.1 & 0.33 & & & \\
\hline 17 & 2 & 15 & 417 & 1 & 16 & 42629.13 & 0.25 & & & \\
\hline 18 & 0 & 18 & +17 & 1 & 17 & 41675.74 & 0.44 & & & \\
\hline 18 & 1 & 17 & +18 & 0 & 18 & 26962.0 & -0.3 & & & \\
\hline 18 & 2 & 16 & +18 & 1 & 17 & 42209.35 & 0.16 & & & \\
\hline 21 & 1 & 20 & +20 & 2 & 19 & 23362.6 & 0.2 & & & \\
\hline 21 & 2 & 19 & $\leftarrow 21$ & 1 & 20 & 41187.86 & -0.33 & & & \\
\hline 22 & 1 & 21 & +21 & 2. & 20 & 2.7259 .8 & -0.1 & & & \\
\hline 22 & 2 & 20 & +22 & 1 & 21 & 40950.62 & -0.14 & & & \\
\hline
\end{tabular}

a. $\Delta v=v_{\text {calc }}-v_{\text {obs }}$ from IAM calculations.

b. Frequencles with one dectmal number are measured as pump setting

only. Frequencles with two decinal numbers are measured with MW-MW.DR unless stated otherwise.

(L) = From Ref (1), (S) = From Stark spectrum.

For b-type lines the average frequency of the quadrupole components is reported. 
computational procedure have been discussed earlier $(14,15)$. In this treatment the possibility of geometry relaxation of molecule is taken into account by introducing the parameters $R_{x x}^{(3)}, R_{y y}^{(3)}, R_{z z}^{(3)}$, and $R_{y z}^{(3)}$.

For 1-chloro-2-butyne ( $E$ type, $J \geqslant 5$ ) the lower $v=1$ levels are found between the higher $v=0$ levels, so we expect considerable mixing between the two states. Therefore, in the final calculations the energy levels were calculated by diagonalizing the full Hamiltonian matrix up to $v=2$, the interactions with higher states being treated by a Van Vleck transformation. Compared to the more crude method, where each torsional state is treated separately and all interactions with the other states are treated by a Van Vleck transformation, some relevant energy levels are shifted by more than $1 \mathrm{GHz}$; the largest effect in an observed transition frequency is over $100 \mathrm{MHz}$. This caused some problems before convergence of the least-square calculations was attained.

The resulting molecular parameters are given in the last column of Table II; the observed rotational frequencies for the first excited state are reported in Table IV. For this calculation the differences $\nu_{\text {calc }}-v_{\text {obs }}$ for the ground state were larger than reported in Table III, the rms deviation for this subset of the data now being $0.76 \mathrm{MHz}$. The inclusion of the $v=1$ data has diminished the correlation coefficient between $I_{\alpha}$ and $A$ from -0.99994 to -0.84 and has allowed us to find $V_{6}$. This parameter is well determined, although its error limits happen to be larger than its value.

Trial calculations showed that the transitions for $v=2$ must be looked for in the same regions as the ground state transitions. In view of the enormous assignment problems the identification of these transitions was not pursued.

TABLE IV

Transition Frequencies and $A, E$ Splittings of the First Excited Torsional State of $\mathrm{CH}_{3} \mathrm{CCCH}_{2} \mathrm{Cl}(\mathrm{MHz})^{\mathrm{b}}$

\begin{tabular}{|c|c|c|c|c|c|c|c|c|c|c|}
\hline \multicolumn{6}{|c|}{ Transition } & \multirow[t]{2}{*}{$v_{A}$} & \multirow[t]{2}{*}{$\Delta v_{A}{ }^{a}$} & \multirow{2}{*}{$\frac{\nu_{E}}{23815.3}$} & \multirow{2}{*}{$\frac{\Delta v_{\mathrm{E}}{ }^{\mathrm{a}}}{0.0}$} & \multirow[t]{2}{*}{$v_{A^{-1}}^{-v_{E}}$} \\
\hline 8 & 1 & 7 & +7 & 1 & 6 & & & & & \\
\hline 8 & 2 & 6 & 7 & 2 & 5 & 24309.8 & 0.3 & & & \\
\hline 9 & 0 & 9 & 8 & 0 & 8 & 23961.4 & -0.5 & 24954.1 & 0.1 & -992.7 \\
\hline 9 & 1 & 9 & $\leftarrow 8$ & 1 & 8 & 23961.4 & -0.4 & 25341.9 & 2.4 & -1380.5 \\
\hline 9 & 1 & 8 & +8 & 1 & 7 & & & 26738.11 & 1.39 & \\
\hline 9 & 2 & 7 & +8 & 2 & 6 & 27055.32 & -0.72 & & & \\
\hline 10 & 0 & 10 & +9 & 0 & 9 & 26854.26 & 0.47 & 27819.09 & -0.49 & -964.83 \\
\hline 10 & 1 & 10 & +9 & 1 & 9 & 26855.04 & -0.11 & 28108.95 & 0.20 & -1253.91 \\
\hline 10 & 1 & 9 & +9 & 1 & 8 & 27533.91 & 0.74 & 29628.93 & 2.63 & -2095.02 \\
\hline 10 & 2 & 8 & +9 & 2 & 7 & 29763.04 & -0.25 & & & \\
\hline 11 & 0 & 11 & +10 & 0 & 10 & 29751.54 & 0.74 & 30689.17 & -0.90 & -937.63 \\
\hline 11 & 1 & 11 & +10 & 1 & 10 & 29751.93 & 0.65 & 30885.42 & -0.73 & -1133.49 \\
\hline 11 & 1 & 10 & +10 & 1 & 9 & 30306.58 & 0.94 & & & \\
\hline 12 & 0 & 12 & +11 & 0 & Il & $32651.68(\mathrm{~s})$ & 0.55 & $33562.17(\mathrm{~s})$ & -0.98 & -910.49 \\
\hline 13 & 0 & 13 & +12 & 0 & 12 & $35553.08 \ldots$ & 0.51 & & & \\
\hline 14 & 0 & 14 & +13 & 0 & 13 & $38455.36 "$ & 0.19 & $39312.72(\mathrm{~s})$ & -2.07 & -857.36 \\
\hline 15 & 0 & 15 & $\leftarrow 14$ & 0 & 14 & $41357.00 "$ & 0.45 & & & \\
\hline
\end{tabular}

a. $\Delta v=v_{\text {calc }}{ }^{-v_{\text {obs }}}$ from IAM calculations with geometry relaxation

(Iast column of Table II).

b, S: see footnotes Table III. 


\section{DISCUSSION}

Table I shows that for the $A$-type transitions of the ground vibrational state a rigid rotor fit, with centrifugal distortion corrections for higher $J$ values, is quite satisfying even for a molecule with a barrier as low as $10 \mathrm{~cm}^{-1}$. Of course, the rotational constants do not represent the molecular structure even approximately, as shown by comparison of the various values for the rotational constant $A$ in Table I and II; indeed, it was just this difference that enabled Laurie and Lide $(l)$ to estimate an upper limit to the barrier height.

The internal axis method used in this work is rather different from the approach used in most other studies on molecules with low internal rotation barriers, as discussed in the Introduction. The method appears to work quite well, and we conclude that the less satisfactory results reported for acetamide (5) could be due to a breakdown of the rigid frame-rigid top approximation rather than to an inadequacy of the IAM for low barriers. In this respect 1-chloro-2-butyne is a favorable case. Moreover, the sixfold contribution to the barrier is negligible within the error limit of $0.3 \mathrm{~cm}^{-1}$. Thus we have not been troubled with ambiguities in the simultaneous determination of $V_{3}$ and $V_{6}$, as encountered in Refs. (3) and (4).

Elementary theory of the chemical bond suggests essentially free rotation about a $-\mathrm{C} \equiv \mathrm{C}-$ axis. The present work shows that the introduction of a chlorine atom into 2-butyne doubles the barrier height $V_{3}$, but the value remains small: $V_{3}=10.2 \pm 0.2$ $\mathrm{cm}^{-1}$ summarizes the results of Table II. Obviously a study of a 1,4 disubstituted 2butyne would be of interest: one expects the barrier to be considerably higher, but still low enough to make the study of internal rotation with an asymmetric internal rotor quite challenging.

RECEIVED: December 19, 1986

\section{REFERENCES}

1. V. W. Laurie AND D. R. Lide, Jr., J. Chem. Phys. 31, 939-943 (1959).

2. R. G. LetT AND W. H. Flygare, J. Chem. Phys. 47, 4730-4750 (1967).

3. H. D. RUDOLPH AND A. TRINKAUS, Z. Naturforsch. A 23, 68-76 (1968).

4. P. H. TuRner, M. J. Corkill, AND A. P. Cox, J. Phys. Chem. 83, 1473-1482 (1979).

5. T. Kojima, E. Yano, K. NaKagaWa, and S. TsuneKaWA, J. Mol. Spectrosc. 112, 494-495 (1985).

6. J. KopuT, J. Mol. Spectrose. 106, 12-21 (1984); 115, 131-146 (1986).

7. J. KopUT, J. Mol. Spectrosc. 118, 189-207 (1986).

8. J. Nakagawa, M. Ilayashi, Y. Endo, S. Saito, and E. Hirota, J. Chem. Phys. 80, 5922-5925 (1984).

9. C. C. Lin And J. D. Swalen, Rev. Mod. Phys. 31, 841-892 (1959).

10. J. Nakagawa, K. Yamada, M. Bester, and G. WInNewisser, J. Mol. Spectrosc. 110, 74-85 (1985).

11. R. D. MCLaChLAN, Spectrochim. Acta A 23, 1793-1801 (1967).

12. B. P. VAN EuCK AND V. M. STOLWIJK, $J$. Mol. Spectrosc. 111, 164-172 (1985).

13. B. P. van Euck, J. van OPheusden, M. M. M. van Schaik, and E. van Zoeren, J. Mol. Spectrosc. 86, 465-479 (1981).

14. B. P. VAN EuCK AND F. B. VAN DUIJNeveldT, J. Mol. Spectrosc. 102, 273-288 (1983).

15. B. P. van Eijck, A. Dubrulle, J. Demaison, ANd J. L. Ripoll, J. Mol. Spectrosc. 112, 95-103 (1985). 